\section{Concentrações de vitamina A no leite humano e características socioeconômicas e nutricionais maternas: resultados de estudos brasileiros}

\section{Vitamin A in human milk and socio- economic and maternal nutritional factors: some results of Brazilian studies}

Julicristie Machado de Oliveira 1

Natália Sanchez Oliveira 2

Denise Pimentel Bergamaschi 3

1-3 Faculdade de Saúde Pública. Universidade de São Paulo. Av. Dr. Arnaldo, 715. São Paulo, SP, Brasil. CEP: 01.246-904. E-mail: denisepb@usp.br

\begin{abstract}
Objectives: to compile the results of Brazilian studies that investigated the vitamin A content of human milk.

Sources: the searches were performed at LILACS, Banco de Teses da Capes, SciELO (Scientific Electronic Library), and Plataforma Lattes databases. The search strategy was: pregnant, lactating, human milk vitamin A concentration, Brazil. The searches were done in 2006 and updated in March 2008.

Summary of the findings: fourteen studies published between 1988 and 2008 were located. These studies were heterogeneous in terms of sample size, milk phase, time of collection and method for determining vitamin A concentrations. The study outcomes described average vitamin A concentrations in human milk ranging from 0.62 to $4.50 \mu \mathrm{mol} / \mathrm{L}$.

Conclusions: there was no consensus as to the relationship between the vitamin A content of human milk and a diet with a suitable vitamin A content, maternal nutritional status, maternal obstetric and demographical characteristics and duration of pregnancy. The review indicates that future studies should use casual samples of mature milk, and use high performance liquid chromatography - HPLC - as the laboratory technique in order to quantify vitamin $A$.
\end{abstract}

Key words Vitamin A, Milk, human, Vitamin A deficiency, Breast feeding

\section{Resumo}

Objetivos: agregar e discutir os resultados de estudos realizados no Brasil que avaliaram a concentração de vitamina A no leite materno.

Fontes dos dados: foram pesquisadas as bases LILACS, Banco de Teses da Capes, SciELO (Scientific Electronic Library), e Plataforma Lattes - seção de produção cientifica. As palavras-chaves utilizadas foram: gestantes, lactante, concentração de vitamina A no leite humano, Brasil. As buscas foram realizadas em 2006 e atualizadas em março de 2008. Foram incluidos todos os estudos localizados.

Sintese dos dados: foram localizados 14 estudos, publicados entre 1988 e 2008, heterogêneos quanto ao tamanho da amostra, fase do leite, período do dia da coleta e método de determinação das concentrações de vitamina A. Foram descritas concentrações médias de vitamina A no leite humano entre 0,62 e $4,50 \mu \mathrm{mol} / \mathrm{L}$.

Conclusões: não houve consenso sobre a relação entre concentração de vitamina A no leite humano e vitamina A dietética, estado nutricional materno, características obstétricas e demográficas e duração da gestação. Sugere-se que estudos futuros utilizem, amostras de leite maduro, coletadas aleatoriamente ao longo dos diferentes periodos do dia, e a utilização do high performance liquid chromatography - HPLC - como método de determinação de vitamina A.

Palavras-chave Vitamina A, Leite humano, Deficiência de vitamina A, Aleitamento materno 


\section{Introdução}

O termo vitamina A abrange um grupo de substâncias lipossolúveis que desempenham atividades biológicas semelhantes no metabolismo humano. 1-3 Estas estão relacionadas à expressão gênica, ao adequado funcionamento do sistema visual, ao crescimento e desenvolvimento físico, à manutenção da integridade das células epiteliais e à função imunológica. Apresentam, ainda, papel fundamental na gestação e lactação, considerando-se as demandas maternas, fetais e do lactente. ${ }^{1-3}$

As fontes dietéticas de vitamina A são alguns produtos de origem vegetal, ricos em pró-vitamina A, e produtos de origem animal, como o fígado, ricos em vitamina A pré-formada. ${ }^{2-4} \mathrm{O}$ leite humano é um exemplo de alimento fonte desse micronutriente.2,4 Quanto à composição do leite materno, há evidências de que teores de proteínas e carboidratos podem sofrer mudanças de acordo com o período de lactação, não variando, de maneira importante, de mulher para mulher. Em contrapartida, a fração lipídica e alguns micronutrientes, entre eles a vitamina A, podem variar entre indivíduos e populações. ${ }^{1}$

A concentração de vitamina A é muito alta no colostro (até o sexto dia pós-parto), continuando alta na fase de transição (7o ao 210 dia pós-parto), e tornando-se estável na fase de leite maduro (após o $21^{\circ}$ dia pós-parto). Este último é o mais apropriado para investigação de deficiência de vitamina A (DVA) subclínica no grupo materno-infantil, por meio da análise do leite humano. 5 Segundo Stoltzfus e Underwood, 6 a concentração de vitamina A no leite humano é um indicador que provê informações que podem estar relacionadas tanto ao estado nutricional materno, quanto ao infantil.

As reservas de vitamina A do recém-nascido podem ser influenciadas pelo estado nutricional da mulher durante e anterior à gestação; assim bebês de gestantes com deficiência desse micronutriente podem nascer com estoque restrito. Da mesma forma, o teor de vitamina A no leite humano poderá depender do estado nutricional materno, principalmente no que diz respeito a esse micronutriente. ${ }^{1}$ Deve-se considerar também que deficiência de zinco, desnutrição materna e situações que ativam uma resposta de fase aguda podem limitar o transporte de vitamina A para o leite materno. ${ }^{7}$

O objetivo do presente estudo foi agregar e discutir os resultados de estudos realizados no Brasil, que avaliaram a concentração de vitamina A no leite materno.

\section{Fontes dos dados}

Foi realizada uma revisão da literatura a partir de palavras-chave, em português e inglês, referentes à população de estudo (gestante, lactante), ao objeto de interesse (concentração de vitamina A no leite humano) e país de realização (Brasil). Foram pesquisadas as bases de dados: LILACS, Banco de Teses da Capes, SciELO (Scientific Electronic Library), e Plataforma Lattes - seção de produção científica. As buscas foram realizadas em 2006 e atualizadas em março de 2008 e todos os estudos que cumpriram os critérios acima especificados foram incluídos, independentemente do ano em que foram publicados.

As concentrações médias de vitamina A foram apresentadas em $\mu \mathrm{mol} / \mathrm{L}$ e, quando disponíveis, em $\mu \mathrm{g} / \mathrm{g}$ de lipídio. Para estudos que apresentaram os dados em $\mu \mathrm{g} / \mathrm{dl}$ ou $\mu \mathrm{gRE} / 100 \mathrm{~mL}$, foram realizadas conversões por meio do Conversor.xls, 8 utilizandose as seguintes equivalências: $1 \quad \mathrm{RE}=1 \mu \mathrm{g} ; 1$ $\mathrm{RE}=0,0034911 \mu \mathrm{mol} ; 1 \mathrm{RE}=3,33 \mathrm{UI}$. Para o $\beta$ caroteno, foram adotados os fatores de conversão sugeridos pelo International Vitamin A Consultative Group (IVACG). 9 Demais conversões foram realizadas no programa Excel.

Adotou-se, como recomendado pela World Health Organization (WHO), 5 concentração de vitamina $\mathrm{A} \leq 1,05 \mu \mathrm{mol} / \mathrm{L}$ ou $\leq 8,0 \mu \mathrm{g} / \mathrm{g}$ de lipídio, como indicador de DVA no leite materno. Consideraramse, como descrito na literatura, 10 concentrações de vitamina A no leite inferiores a $1,75 \mu \mathrm{mol} / \mathrm{L}$ como insuficientes para a constituição de reservas hepáticas adequadas de vitamina A pelos recémnascidos.

Ainda, seguindo recomendação da WHO para identificação de DVA subclínica materna ou infantil, utilizou-se concentração sérica de vitamina $\mathrm{A} \leq 0,7$ $\mu \mathrm{mol} / \mathrm{L} .5$

Segundo o Institute of Medicine (IOM), constituem valores de referência para ingestão diária (Recommended Dietary Allowances - RDA) de vitamina A, para gestantes, $770 \mu \mathrm{gRE}$, e para lactantes, $1300 \mu \mathrm{gRE} / \mathrm{dia}^{4}$

Optou-se por apresentar nesta revisão valores de concentração de vitamina A em quantidades médias, desvios-padrão (DP), intervalos de confiança de 95\% utilizando-se a distribuição t de "Student" e coeficientes de variação Pearson. 


\section{Síntese dos Dados}

Foram localizados 14 estudos, publicados entre 1988 e 2008, que descreveram as concentrações de vitamina $A$ e/ou e $\beta$-caroteno no leite humano, sendo nove divulgados em formato de artigos científicos 11 19 e cinco como trabalhos de conclusão de mestrado ou doutorado. $20-24$

A Tabela 1 apresenta características metodológicas dos estudos analisados. Observa-se que são heterogêneos em relação ao tamanho da amostra estudada, fase do leite (colostro, transição e maduro), período do dia de coleta, micronutrientes avaliados e método de determinação de vitamina A. Os estudos, em grande parte $(n=8,57 \%)$, foram realizados com amostras inferiores a 50 nutrizes. Somente em dois houve descrição do cálculo do tamanho amostral.17,18 Os estudos foram predominantemente observacionais, sendo somente um conduzido com desenho experimental.17 Quanto à fase do leite, dez artigos utilizaram apenas colostro ou leite maduro ( $n=6$ e 4 , respectivamente), enquanto três analisaram duas $(\mathrm{n}=2)$ ou as três fases $(\mathrm{n}=1)$. Apenas um estudo não mencionou qual foi o período de lactação referente à coleta das amostras de leite.

Como método de determinação do teor de vitamina A ou $\beta$-caroteno, foi utilizado, em 12 estudos, o HPLC (High Performance Liquid Chromatography) e, nos demais, foi utilizada a espectrofotometria.

As concentrações médias de vitamina A em estudos que utilizaram o leite maduro variaram entre 0,62 e $2,53 \mu \mathrm{mol} / \mathrm{L}$. Apesar dessa variação, pode-se sugerir que há uma semelhança entre os valores médios, se consideradas as sobreposições dos intervalos de confiança, com exceção dos estudos que apresentam o maior11 e menor nível médio. 16 Para o colostro, a média da concentração de vitamina A no leite humano, excluindo-se o grupo de recémnascidos pré-termo, variou de 2,48 a $4,50 \mu \mathrm{mol} / \mathrm{L}$, porém houve sobreposição dos valores dos intervalos de confiança (Tabela 1), o que indica ausência de diferença estatisticamente significativa.

Comparando-se os valores mínimo e máximo de concentração de vitamina A no leite, observou-se que mesmo nos estudos onde as concentrações médias estavam acima do ponto de corte, parte dos valores estavam abaixo desse teor, indicando uma possível situação de DVA subclínica. Na Tabela 1, pode-se observar prevalências importantes de concentrações abaixo de $1,05 \mu \mathrm{mol} / \mathrm{L}$.

Somente três estudos apresentam dados de concentração de vitamina $\mathrm{A}$ em $\mu \mathrm{g} / \mathrm{g}$ de lipídio (Tabela 2). Apesar do nível médio de vitamina A estar acima do ponto de corte nos dois estudos, os valores mínimos indicam que parte das nutrizes apresentava-se deficiente em vitamina A. As concentrações de $\beta$-caroteno no leite humano foram dosadas em três estudos, com resultados de teores semelhantes (Tabela 3).

A vitamina A dietética do grupo materno foi avaliada em seis estudos, sendo que em três deles investigou-se a correlação entre essa variável e a concentração da vitamina no leite humano. A adequação do consumo de vitamina $\mathrm{A}$ (em retinol equivalente) pelas mulheres variou de $22 \%$ a $55 \%$, como pode ser observado na descrição dos estudos.

Vítolo et al.11 investigaram a vitamina $\mathrm{A}$ dietética das nutrizes segundo faixa etária e condição socioeconômica (CS). Os resultados mostram valores médios diários menores, porém não estatisticamente significantes $(p=0,07)$, para as adolescentes de baixa CS ( $\mathrm{n}=26 ; 281,5 \mu \mathrm{gRE} / \mathrm{dia})$, quando comparadas com os grupos de nutrizes adultas de baixa CS ( $\mathrm{n}=21 ; 412,1 \mu \mathrm{gRE} / \mathrm{dia})$ e alta $\mathrm{CS}(\mathrm{n}=27$; 770,4 $\mu \mathrm{gRE} / \mathrm{dia})$.

Presta22 relatou inadequação do teor dietético de vitamina A, avaliando o consumo alimentar das nutrizes por meio de questionário de freqüência alimentar (QFA) e recordatório de 24h (R24). Segundo dados obtidos pelo QFA, 78\% das nutrizes apresentaram ingestão abaixo das recomendações, com valor médio de 1214 ( $\mathrm{DP}=840 \mu \mathrm{gRE} / \mathrm{dia})$. A ingestão média obtida pelo recordatório de $24 \mathrm{~h}$ foi de 1174 (DP=1771 $\mu \mathrm{gRE} /$ dia), também se mostrando abaixo do recomendado (1300 $\mu \mathrm{gRE} / \mathrm{dia})$.

Dimenstein et al.12 relataram que $55 \%$ das mulheres apresentavam teor dietético de vitamina A adequado ao final da gestação, com média de ingestão de 1398,8 $\mu \mathrm{gRE} / \mathrm{dia}$, e teores inadequados para as demais (45\%). Contudo, no estudo de Passos, $2030 \%$ das mulheres apresentaram vitamina A dietética adequada segundo as recomendações para gestantes. Em seu estudo, a média de vitamina A na dieta variou de 527 a $1376 \mu \mathrm{gRE} / \mathrm{dia}$, com valor médio de 781 ( $\mathrm{DP}=247 \mu \mathrm{gRE} / \mathrm{dia})$.

Nas investigações conduzidas por $\operatorname{Passos}^{20} \mathrm{e}$ Presta, 22 não foram encontradas correlações estatisticamente significantes entre a vitamina A dietética e as concentrações de vitamina A no leite materno. Para o $\beta$-caroteno, Presta 22 observou correlação estatisticamente significante entre a ingestão habitual e as concentrações no leite $(\mathrm{r}=0,32 ; p=0,05)$.

Dimenstein et al. 19 observaram baixo consumo de vitamina A em $35,9 \%$ das mulheres avaliadas. A média de ingestão, estimada a partir de um QFA, foi de 1737,6 (1785,1) $\mu$ gRAE/dia. Esses autores relataram também correlação positiva e estatisticamente significativa entre a vitamina A dietética e a 


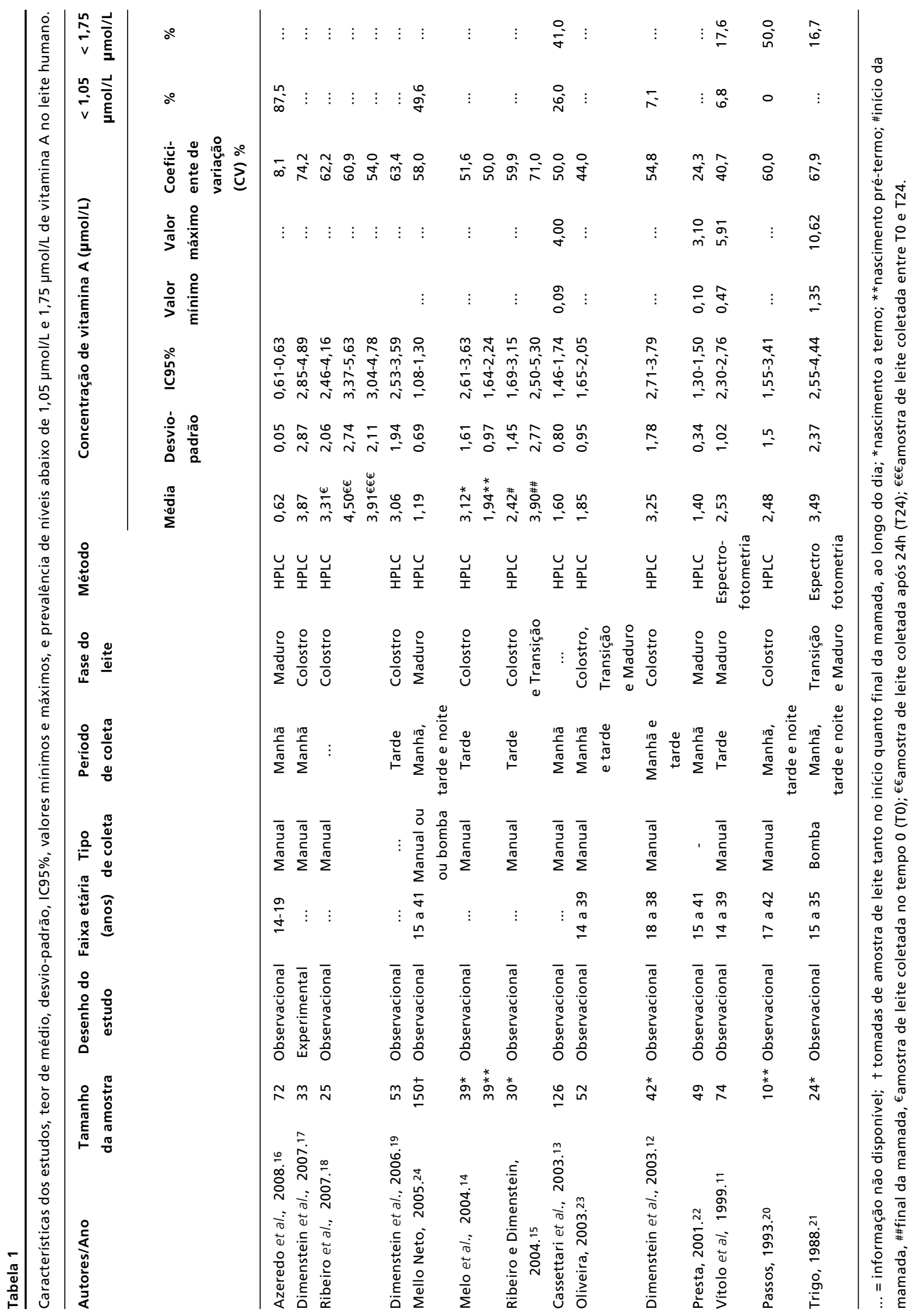




\begin{tabular}{|c|c|c|c|c|c|}
\hline \multirow[t]{2}{*}{ Autores/Ano } & \multirow{2}{*}{$\begin{array}{l}\text { Tamanho da } \\
\text { amostra (n) }\end{array}$} & \multicolumn{2}{|c|}{ Teor de vitamina A ( $\mu \mathbf{g} / \mathbf{g}$ de lipídio) } & \multirow[t]{2}{*}{ Valores mínimos e máximos } & \multirow[t]{2}{*}{$\%<8 \mu \mathrm{g} / \mathrm{g}$ de lipídio } \\
\hline & & Valor médio & Desvio padrão & & \\
\hline Presta, 2001.22 & 40 & 14,32 & 18,05 & 2,86 a 60,15 & $\ldots$ \\
\hline Cassettari et al., 2003.13 & 126 & 12,40 & 7,30 & 0,65 a 45,4 & 28,00 \\
\hline Ribeiro et al., $2007 . € 18$ & 25 & 43,0 & 31,9 & $\ldots$ & $\ldots$ \\
\hline
\end{tabular}

$€$ Amostra de leite entre $T_{0}$ e $T_{24}$.

Tabela 3

\begin{tabular}{lcccc}
\hline Teor médio, desvio-padrão, valores mínimos e máximos de $\beta$ - caroteno no leite humano ( $\mu \mathrm{mol} / \mathrm{L})$. & \\
\hline Autores/Ano & Tamanho da amostra $(\mathrm{n})$ & Teor de $\beta$-caroteno $(\mu \mathrm{mol} / \mathrm{L})$ & $\begin{array}{c}\text { Valores mínimos e máximos } \\
(\mu \mathrm{mol} / \mathrm{L} \text { de } \beta \text {-caroteno) }\end{array}$ \\
\cline { 3 - 5 } & & Valor médio & Desvio padrão & \\
\hline Passos, 1993.20 & 10 & 0,011 & 0,007 & traço a 0,020 \\
Presta, 2001.22 & 38 & 0,018 & 0,014 & 0,003 a 0,063 \\
Azeredo et al. 2008.16 & 72 & 0,016 & 0,002 & $\ldots$
\end{tabular}

$€$ Amostra de leite entre $T_{0}$ e $T_{24}$.

concentração de vitamina A no soro das mulheres $(\mathrm{r}=0,43 ; p=0,04)$ e a concentração de vitamina A no colostro $(\mathrm{r}=0,49 ; p=0,03)$.

No estudo conduzido por Vítolo et al.,11 foram encontradas concentrações médias de vitamina A no leite humano de $2,48 \mu \mathrm{mol} / \mathrm{L}(\mathrm{DP}=1,06 \mu \mathrm{mol} / \mathrm{L}) \mathrm{e}$ $2,85 \mu \mathrm{mol} / \mathrm{L}(\mathrm{DP}=1,14 \mu \mathrm{mol} / \mathrm{L})$ para nutrizes adolescentes e adultas de baixa condição socioeconômica, respectivamente. Tais médias, quando comparadas aos valores médios para nutrizes adultas de alta CS, $2,31 \mu \mathrm{mol} / \mathrm{L}(\mathrm{DP}=0,84 \mu \mathrm{mol} / \mathrm{L})$, não apresentaram diferenças estatisticamente significantes $(p=0,23)$. Dimenstein et al. 12 corroboram os achados anteriores, descrevendo médias de vitamina A no colostro para nutrizes de baixa e alta renda de $3,47 \mu \mathrm{mol} / \mathrm{L}$ $(\mathrm{DP}=1,91 \mu \mathrm{mol} / \mathrm{L})$ e $2,98 \mu \mathrm{mol} / \mathrm{L}(\mathrm{DP}=1,63 \mu \mathrm{mol} / \mathrm{L})$, respectivamente $(p=0,503)$. Os autores investigaram também a relação entre nível de escolaridade e concentração de vitamina A no leite, sem evidência estatística de diferenças. A ausência de associação também foi descrita por Trigo, ${ }^{21}$ ao estudar a relação entre as concentrações de vitamina A no leite e área de moradia, com estimativas de média de vitamina A no leite humano de moradoras de área urbana pobre e de classe média de $4,20 \mu \mathrm{mol} / \mathrm{L}$ ( $\mathrm{DP}=3,14 \mu \mathrm{mol} / \mathrm{L}$ ) e $2,78 \mu \mathrm{mol} / \mathrm{L}(\mathrm{DP}=0,89 \mu \mathrm{mol} / \mathrm{L})$, respectivamente $(p=0,52)$.

Dimenstein et al.,12 investigaram a relação entre a concentração de vitamina A no colostro e o estado nutricional da mulher durante a gestação, e descrevem diferenças estatisticamente significantes $(p=0,016)$ entre mulheres com sobrepeso (teor médio: 4,07 $\mu \mathrm{mol} / \mathrm{L}$; IC95\%: $2,8-5,34 \mu \mathrm{mol} / \mathrm{L})$, eutróficas (teor médio: $3,34 \mu \mathrm{mol} / \mathrm{L}$; IC95\%: 2,6 $4,08 \mu \mathrm{mol} / \mathrm{L}$ ), e com baixo peso (teor médio: 2,21 $\mu \mathrm{mol} / \mathrm{L}$; IC95\%: 1,32 - 3,10 $\mu \mathrm{mol} / \mathrm{L})$. Presta, 22 investigou a distribuição conjunta do teor de vitamina A do leite humano e o Índice de Massa Corporal (IMC) materno, e encontrou correlação positiva estatisticamente significante $(\mathrm{r}=0,32$; $p=0,04)$, indicando aumento da concentração de vitamina A com aumento do IMC.

Parece não haver consistência na literatura pesquisada sobre a relação positiva entre a concentração de vitamina A no leite e estado nutricional materno se for considerado o estudo de Oliveira, 23 que também utilizou o IMC e a classificação do estado nutricional de nutrizes em três categorias 
(desnutridas; normais; sobrepeso e obesidade) e encontrou evidências estatísticas de maior concentração de vitamina A no colostro de mulheres classificadas como normais do que entre as obesas. Ainda, para o leite de transição, as que tiveram IMC abaixo de $18,5 \mathrm{~kg} / \mathrm{m}^{2}$ apresentaram maior teor médio que as mulheres das outras duas categorias, porém somente quatro nutrizes foram classificadas nessa categoria.

Somente três do total de artigos incluídos na presente revisão investigaram a relação entre nascimento com baixo peso ou pré-termo com a concentração de vitamina A no leite materno. Os achados desses estudos não são consistentes quanto à relação entre a concentração de vitamina A no leite e o peso ao nascer ou nascimento pré-termo. Enquanto Dimenstein et al.12 não encontram evidência estatística de correlação, apresentando valores médios de vitamina $A$ iguais a $2,67 \mu \mathrm{mol} / \mathrm{L}(\mathrm{DP}=1,90 \mu \mathrm{mol} / \mathrm{L})$ e $3,31 \mu \mathrm{mol} / \mathrm{L}(\mathrm{DP}=1,81 \mu \mathrm{mol} / \mathrm{L})$ entre recémnascidos de baixo peso e de peso adequado, respectivamente, Melo et al.14 descrevem valores médios menores $(p=0,001)$ entre recém-nascidos pré-termo (1,94 $\mu \mathrm{mol} / \mathrm{L})$, quando comparados aos nascidos a termo $(3,12 \mu \mathrm{mol} / \mathrm{L})$ (Tabela 1). Resultado diferente foi encontrado por Oliveira, 23 que descreve valores médios de vitamina A maiores no colostro de mães de recém-nascidos pré-termo $(3,57 \mu \mathrm{mol} / \mathrm{L}$; IC95\%: $2,55-4,59 \mu \mathrm{mol} / \mathrm{L})$ em relação aos bebês nascidos a termo $(2,11 \mu \mathrm{mol} / \mathrm{L}$; IC95\%: $1,77-2,45 \mu \mathrm{mol} / \mathrm{L})$.

A relação entre a concentração de vitamina $A$ plasmática e no leite humano foi investigada por Presta, 22 com estimativa de média de vitamina A plasmática de $1,7(0,68) \mu \mathrm{mol} / \mathrm{L}$, e valores mínimo e máximo iguais a 0,5 e $3,3 \mu \mathrm{mol} / \mathrm{L}$, indicando que algumas mulheres apresentavam DVA subclínica ( $2 \%$ de prevalência de concentração plasmática $<0.7$ $\mu \mathrm{mol} / \mathrm{L})$. Azeredo et al. 16 investigaram a concentração plasmática de vitamina $\mathrm{A}$ de mães adolescentes e descrevem média de 2,1 $(0,06) \mu \mathrm{mol} / \mathrm{L}$. Em ambos estudos, investigou-se a correlação entre a concentração de vitamina A plasmática e no leite humano, porém não foram observadas diferenças significativas.

Somente três estudos investigaram a relação entre a concentração de vitamina A no leite humano e as características demográficas e obstétricas maternas. Presta 22 observou influência da paridade na concentração de vitamina A no leite, com maiores médias entre as multíparas se comparadas às primíparas $(p=0,03)$. Estes resultados, entretanto, não estão em acordo aos apresentados por outros autores. Trigo 21 descreve ausência de correlação entre a concentração de vitamina A no leite e paridade para mulheres tanto de áreas urbanas de classe média e pobre. Resultado semelhante foi descrito por Oliveira $^{23}$ ao analisar as três fases de lactação.

Em relação à influência da idade materna, foi observada, no estudo conduzido por Mello Neto, ${ }^{24}$ correlação estatisticamente significante entre essa variável e a concentração de vitamina A no leite humano $(\mathrm{r}=0,18 ; p=0,032)$. $\mathrm{O}$ autor também relata correlação negativa estatisticamente significante entre o tempo de amamentação e a concentração dessa vitamina no leite materno $(\mathrm{r}=-0,28 ; p=0,001)$.

$\operatorname{Presta}^{22}$ descreveu existência de correlações positivas e estatisticamente significantes entre as concentrações de vitamina $A$ e $\beta$-caroteno $(r=0,61$; $p=0,002)$, vitamina A e luteína/zeaxantina $(\mathrm{r}=0,38$; $p=0,02)$ e vitamina A e lipídio $(\mathrm{r}=0,44 ; p=0,006)$ no leite materno. Mello Neto24 relata correlação estatisticamente significativa entre as concentrações de vitamina A e de ferro no leite humano $(\mathrm{r}=0,18$; $p=0,030)$. Azeredo et al. 16 observaram correlações significativas entre vitamina $\mathrm{A}$ e $\beta$-caroteno $(\mathrm{r}=0,63$; $p<0,001), \quad \alpha$-caroteno $(\mathrm{r}=0,35 ; p=0,015)$ e $\alpha$ tocoferol $(0.41, p=0.004)$ no leite humano.

Cinco estudos analisaram as variações nas concentrações de vitamina A no leite. Passos 20 investigou a composição do leite humano de mães de recém-nascidos pré-termo e, para uma sub-amostra de quatro mulheres, apresentou concentrações de vitamina A no colostro e no leite de transição (15 dias pós-parto), com médias iguais a 3,63 $\mu \mathrm{mol} / \mathrm{L}$ $(\mathrm{DP}=1,78 \mu \mathrm{mol} / \mathrm{L})$ e $1,50 \mu \mathrm{mol} / \mathrm{L} \quad(\mathrm{DP}=0,59$ $\mu \mathrm{mol} / \mathrm{L})$, para o colostro e o leite de transição, respectivamente. Os dados obtidos por Oliveira 23 indicaram concentrações médias de vitamina A maiores no colostro $(2,62 \mu \mathrm{mol} / \mathrm{L} ; \quad \mathrm{DP}=1,17$ $\mu \mathrm{mol} / \mathrm{L})$, quando comparados ao leite de transição $(1,84 \mu \mathrm{mol} / \mathrm{L} ; \mathrm{DP}=0,09 \mu \mathrm{mol} / \mathrm{L})$ e ao maduro $(1,47$ $\mu \mathrm{mol} / \mathrm{L} ; \mathrm{DP}=0,06 \mu \mathrm{mol} / \mathrm{L})$, sem identificar diferença entre as médias das duas últimas fases $(p=0,000)$.

A influência do período do dia (manhã, tarde ou noite) nas concentrações de vitamina A do leite materno foi investigada por Trigo, ${ }^{21}$ com indicação de tendência de aumento nas concentrações médias nas frações de leite no decorrer do dia. Ribeiro e Dimenstein 15 observaram que o conteúdo de vitamina A no leite variou, inclusive, quando comparadas as concentrações de vitamina A ao início (2,42 $\mu \mathrm{mol} / \mathrm{L})$ e ao final $(3,90 \mu \mathrm{mol} / \mathrm{L})$ de uma mesma mamada, sendo essa diferença estatisticamente significante $(p=0,012)$.

Azeredo et al. 16 investigaram a influência do tipo de aleitamento materno (exclusivo e predominante) nas concentrações de vitamina A no leite, porém não encontraram diferenças significativas.

Ribeiro et al.18 pesquisaram a variação da 
concentração de vitamina A no leite humano durante 24 horas. Foram coletadas três amostras, sendo uma no tempo 0 (T0), uma intermediária e uma no tempo 24 (T24). Os resultados estão apresentados na Tabela 1. Houve diferenças estatisticamente significativas entre as concentrações de vitamina A expressas por $\mu \mathrm{mol} / \mathrm{L}$ de acordo com o momento de coleta $(p<0,05)$. Porém, quando expressas por $\mu \mathrm{g} / \mathrm{g}$ de lipídio no leite, essas diferenças não foram significativas: $\mathrm{T}_{0}(44,2 \mu \mathrm{g} / \mathrm{g}$; $\mathrm{DP}=38,4 \mu \mathrm{g} / \mathrm{g})$; intermediária $(46,8 \mu \mathrm{g} / \mathrm{g} ; \mathrm{DP}=38,3 \mu \mathrm{g} / \mathrm{g})$ e $\mathrm{T}_{24}(43,0 \mu \mathrm{g} / \mathrm{g}$; $\mathrm{DP}=31,9 \mu \mathrm{g} / \mathrm{g})$.

Somente um dos estudos revisados avaliou o impacto da suplementação com vitamina A direcionada a mulheres no pós-parto (dose de 200.000 IU). 17 Houve aumento significativo na concentração de vitamina A no leite humano seis horas após a suplementação (antes: $3,87 \mu \mathrm{mol} / \mathrm{L} ; \quad \mathrm{DP}=2,87$ $\mu \mathrm{mol} / \mathrm{L}$ vs após: $5,74 \mu \mathrm{mol} / \mathrm{L} ; \mathrm{DP}=3,72 \mu \mathrm{mol} / \mathrm{L}$, $p<0,025)$. Os autores não observaram influência da idade materna e do tipo de parto na resposta à suplementação com vitamina $\mathrm{A}$.

A interpretação dos resultados dos trabalhos investigados encontrou limitações, tais como: a utilização de diferentes métodos de determinação das concentrações de vitamina A no leite, as fases do leite analisadas, os pontos de corte para identificação de DVA subclínica, além dos diferentes tamanhos de amostras, desenhos metodológicos, ausência da descrição da história obstétrica materna bem como do método de seleção da amostra na maior parte dos estudos. Essas limitações dificultaram a comparação e a análise dos dados, uma vez que não se pode inferir se as diferenças encontradas entre os estudos são reais ou são produtos de características metodológicas diferentes.

Apesar da influência da vitamina A dietética nas concentrações dessa vitamina no leite humano ter sido investigada nos estudos revisados por meio de diferentes métodos de inquérito alimentar com análises realizadas em softwares distintos, os resultados foram semelhantes ao relatarem uma proporção importante de vitamina A dietética abaixo do recomendado, tanto em gestantes como em lactantes. Esses resultados foram semelhantes aos resultados descritos por estudos conduzidos em países em desenvolvimento, sendo a média de ingestão de vitamina A de mulheres não suplementadas $(660 \mu \mathrm{gRE} / \mathrm{dia})$ inferior à metade da encontrada entre lactantes dos países desenvolvidos (1540 $\mu \mathrm{gRE} / \mathrm{dia}) .25 \mathrm{O}$ consumo de vitamina A pré-formada, proveniente de alimentos de origem animal, é muito menor em países em desenvolvimento quando comparado à ingestão de alimentos de origem vegetal, que contêm carotenóides com atividade de pró-vitamina A, principalmente $\beta$-caroteno. Considerando-se que a conversão de $\beta$-caroteno em vitamina A é afetada por uma série de fatores, ${ }^{26}$ é possível que nesses países, além de ingestão dietética de alimentos fontes de vitamina A préformada deficiente, possa haver limitação da absorção e conversão da pró-vitamina A em sua forma ativa.

Somente um estudo incluído na presente revisão investigou a associação entre condições socioeconômicas e vitamina A dietética, porém não foi encontrada diferença significativa. ${ }^{11}$ Nessa investigação, a avaliação do consumo alimentar foi realizada com a aplicação de um recordatório de 24 h, o que pode ser insuficiente para caracterização da ingestão alimentar habitual. Por conseqüência, a observação de diferenças entre vitamina A dietética segundo condições socioeconômicas pode ter sido limitada.

Os resultados dos estudos revisados não são concordantes em relação à associação entre vitamina A dietética e concentrações de vitamina A no leite materno.19,20,22 Por outro lado, tem sido evidenciada que a mudança no estado nutricional relativo à vitamina A em lactantes, após a suplementação com essa vitamina, se reflete de forma importante em aumento de suas concentrações no leite humano.27-30

Evidências do aumento da concentração de vitamina

A no leite em resposta à maior ingestão dietética têm sido descritas em estudos experimentais com animais. Green et al.31 observaram que animais alimentados com dieta isenta de vitamina A apresentam como via exclusiva de transporte da vitamina para o leite a proteína RBP (Retinol Binding Protein). Quando o teor de vitamina A ingerido foi quadruplicado, os quilomícrons passaram a contribuir com $74 \%$ do total de vitamina A transportada para o leite humano, demonstrando seu papel como carreador dessa vitamina para o leite.

Os estudos realizados no Brasil11,12,21 que investigaram a associação entre condição socioeconômica materna e concentração de vitamina A no leite não incluem a descrição do cálculo de tamanho de amostra. Sendo assim, o número de mulheres incluídas ( $\mathrm{n}=24,42$ e 74) nos estudos pode ter sido insuficiente para detecção de diferenças significativas do ponto de vista estatístico, uma vez que o tamanho da amostra interfere no poder do teste de hipóteses.

Além disso, deve-se considerar ainda que no estudo brasileiro conduzido por Dimenstein et al.,12 as participantes apresentavam situação socioeconômica homogênea e baixa, dificultando a identi- 
ficação de associação entre essa variável e a concentração de vitamina A no leite humano.

A condição socioeconômica materna tem sido apontada por estudos internacionais como fator associado à concentração de vitamina A no leite. GebreMedhin et al. 32 encontraram valores maiores e estatisticamente significantes quando comparadas às concentrações médias de vitamina A no leite de mulheres suecas $(1,4 \mu \mathrm{mol} / \mathrm{L}$; IC95\%: 1,19 - 1,62 $\mu \mathrm{mol} / \mathrm{L})$ e de etíopes em condições sociais menos favoráveis $(0,98 \mu \mathrm{mol} / \mathrm{L}$; IC95\%: 0,78 - 1,18 $\mu \mathrm{mol} / \mathrm{L})$, entre 3,5 e 6,5 meses pós-parto. Barua et al.,33 em estudo com nutrizes de Bangladesh, também encontraram maiores concentrações de vitamina A no leite no grupo de maior renda, sendo as concentrações médias no grupo de menor renda 0,99 $\mu \mathrm{mol} / \mathrm{L}(\mathrm{DP}=0,06 \mu \mathrm{mol} / \mathrm{L})$ insuficientes para suprir as recomendações para os lactentes.

Outros fatores, além da deficiência de vitamina A, podem influenciar o estado nutricional relativo a essa vitamina, como a deficiência de zinco ou de ferro. $\mathrm{O}$ estado nutricional relativo ao zinco influencia vários aspectos do metabolismo de vitamina $\mathrm{A}$, incluindo sua absorção, transporte e utilização. 34 Dijkhuisen et al.35 descrevem maior concentração de vitamina A no leite entre mulheres que receberam suplementação combinada de zinco com $\beta$-caroteno (40,7 nmol/g de lipídio) quando comparadas ao grupo placebo $(27,9 \mathrm{nmol} / \mathrm{g}$ de lipídio), no sexto mês pós-parto.

Em estudos em animais e humanos, tem-se descrito a associação entre a deficiência de ferro e baixa concentração sérica de vitamina A.36-41 Shatrugna et al.41 observaram aumento de 0,12 $\mu \mathrm{mol} / \mathrm{L}$ na concentração sérica de vitamina $\mathrm{A}$ em gestantes suplementadas somente com ferro, bem como queda significativa na prevalência de DVA $(20,7 \%)$, indicando eficácia da suplementação isolada de ferro para a melhora do estado nutricional relativo à vitamina $\mathrm{A}$. Apesar da importância da interação entre a vitamina $\mathrm{A}$ e outros micronutrientes, a maior parte dos estudos brasileiros restringiram-se a investigar o estado nutricional relativo somente a essa vitamina, sendo, portanto, interessante agregar também a avaliação do estado nutricional relativo ao ferro e zinco.

Assim como na presente revisão, os dados da literatura internacional são conflitantes quanto à relação entre concentração de vitamina A no leite e duração da gestação 42,43 e a paridade materna. 44,45 Segundo Meneses e Trugo, 46 há possibilidade de que paridade e amamentação anteriores estejam relacionadas a aumento da absorção intestinal, maior captação pela glândula mamária e/ou aumento da mobilização das reservas, o que explicaria as maiores concentrações de vitamina A entre as multíparas.

A ausência de concordância sobre a influência do estado nutricional materno avaliado pelo IMC nas concentrações de vitamina A no leite humano, descrita na presente revisão, também é encontrada na literatura internacional, 44,47 sendo necessários estudos subseqüentes para melhor elucidação da questão.

Estudos incluídos na presente revisão descrevem variação na concentração de vitamina A no leite segundo período do dia (manhã, tarde ou noite) e momento da mamada (início ou final). De acordo com Stoltzfus e Underwood, ${ }^{6}$ há maior concentração de vitamina A no leite de uma mama recentemente sugada, no leite do final de uma mamada e do meio da manhã. Sendo assim, as amostras casuais de leite, retiradas em diferentes momentos do dia e sem controlar quando ocorreu a última amamentação, se constituem em melhor preditor do estado nutricional relativo a essa vitamina. 48 Metade dos estudos brasileiros incluídos na presente revisão utilizou a coleta de amostras durante os diferentes períodos do dia. Assim, pode-se presumir que esses possam fornecer estimativas válidas da concentração média de vitamina A no leite. 12,20,21,23,24

Resultados de estudos metodológicos, que avaliaram a habilidade de diferentes parâmetros, indicam que a concentração de vitamina A no leite humano, expressa por grama de lipídio, constitui-se um dos melhores indicadores do estado nutricional de vitamina A.47,49 Apesar disto, somente três estudos utilizaram o teor de vitamina A expresso por grama de lipídio.13,18,22

Segundo a World Health Organization (WHO), 5 o leite materno maduro secretado a partir do 210 dia pós-parto é o mais adequado para estimar o estado nutricional relativo à vitamina $\mathrm{A}$, uma vez que após esse período a concentração de vitamina A no leite se estabiliza. Na presente revisão, foram identificados quatro estudos que avaliaram a concentração de vitamina A nessa fase do leite.11,16,22,24

Para determinação da vitamina A sérica e no leite, o HPLC é considerado como a melhor metodologia. 50 Pela utilização dessa metodologia em doze estudos brasileiros, $12-20,22-24$ pode-se concluir que provavelmente houve boa quantificação das concentrações desse micronutriente. Os demais estudos11,21 utilizaram espectrofotometria, que, segundo De Pee e Dary, 50 pode apresentar baixa especificidade, baixa acurácia e dificuldades de validação.

A concentração sérica de vitamina A é um indi- 
cador muito utilizado nos estudos que investigam a prevalência de DVA subclínica, porém, como o complexo retinol-RBP é um reactante negativo de fase aguda,51,52 sua interpretação está limitada na presença de inflamação ou infecção. Dancheck et $a l ., 52$ apontaram a ausência de associação entre inflamação, reação de fase aguda e concentração de vitamina A no leite humano. Os autores concluíram que a concentração de vitamina A no leite materno pode ser melhor indicador de DVA subclínica quando comparado à concentração sérica de vitamina $\mathrm{A}$, pois, aparentemente, não sofre influência da reação de fase aguda.

Conclui-se que características, tais como: tamanho adequado do grupo estudado; utilização de

\section{Referências}

1. Butte NF, Lopez-Alarcon MG, Garza C. Nutrient adequacy of exclusive breastfeeding for the term infant during the first six months of life. Expert Consultation on the Optimal Duration of Exclusive Breastfeeding. Geneva: WHO; 2002.

2. FAO (Food and Agriculture Organization of United Nations), who (/World Health Organization). Human vitamin and mineral requirements. Thailand; 2001.

3. WHO (World Health Organization). Safe vitamin A dosage during pregnancy and lactation: recommendations and report of a consultation. Geneva; 1998.

4. IOM (Institute of Medicine). Dietary reference intakes for vitamin A, vitamin $\mathrm{K}$, arsenic, boron, chromium, copper, iodine, iron, manganese, molybdenum, nickel, silicon, vanadium and zinc. Washington, DC: National Academy Press; 2001.

5. WHO (World Health Organization). Indicators for assessing vitamin A deficiency and their application in monitoring and evaluating intervention programmes. Geneva; 1996.

6. Stoltzfus RJ, Underwood BA. Breast milk vitamin A as an indicator of the vitamin A status of women and infants. Bull World Health Organ. 1995; 73: 703-11.

7. Rasmussen K. Vitamin A needs during pregnancy and lactation for the health of the mother and her fetus or infant. In: WHO (World Health Organization). Safe vitamin A dosage during pregnancy and lactation: recommendations and report of a consultation. Geneva; 1998. p. 8-14.

8. Brasil. Ministério da Saúde. Conversor xls. [programa de computador]. 2004. Acesso em 17 jun. 2006]. Disponível em:

dtr2004.saude.gov.br/nutricao/documentos/conversor.xls

9. IVACG (International Vitamin A Consultative Group). Conversions factors for vitamin A and carotenoids. Washington, DC; 2004.

10. Underwood BA. Maternal vitamin A status and its importance in pregnancy and early childhood. Am J Clin Nutr. 1994; 59 (Supl): S517-S24.

11. Vítolo MR, Accioly E, Ramalho RA, Soares AG, Cardoso $\mathrm{CB}$, Carvalho EB. Níveis de vitamina A no leite maduro de nutrizes adolescentes e adultas de diferentes estratos socioeconômicos. Rev Ciênc Med. 1999; 8: 3-10. amostras de leite maduro, coletadas de maneira aleatória e ao longo dos diferentes períodos do dia; utilização de HPLC como método para quantificar as concentrações de vitamina A no leite, utilização do teor expresso em $\mu \mathrm{g} / \mathrm{g}$ de lipídio e investigação de deficiência de ferro e/ou zinco, devem ser consideradas na avaliação do estado nutricional relativo à vitamina A em lactantes.

A utilização do leite humano para detecção de DVA subclínica no grupo materno-infantil deve ser incentivada, pois a coleta do leite não configura em um procedimento invasivo, é bem aceita pelas lactantes, além de haver indícios de que esse indicador possa de maneira mais adequada refletir o estado nutricional relativo a essa vitamina.
12. Dimenstein R, Simplício JL, Ribeiro KDS, Melo ILP. Influência de variáveis socioeconômicas e de saúde materno-infantil sobre os níveis de retinol no colostro humano. J Pediatr. (Rio J.) 2003; 79: 513-8.

13. Cassettari ML, Dias LCG, Giarola LC, Souza N. Determinação de vitamina A no leite materno por cromatografia líquida de alta eficiência (CLAE), Botucatu, SP, Brasil. Nutr Rev Soc Bras Aliment Nutr. 2003; 25: 2330 .

14. Melo ILP, Ribeiro KDS, Dimenstein R Estudos das variações dos níveis de retinol no leite no colostro humano de parturientes a termo e pré-termo. Rev Bras Saúde Matern Infant. 2004; 4: 249-52.

15. Ribeiro KDS, Dimenstein R. Níveis de retinol no leite materno ao início e final da mamada. Rev Panam Salud Publica. 2004; 16: 19-22.

16. Azeredo VB, Trugo NMF. Retinol, carotenoids, and tocopherols in the milk of lactating adolescents and relationships with plasma concentrations. Nutrition. 2008; 24: 1339.

17. Dimenstein R, Lourenço RMS, Ribeiro KDS. Impacto da suplementação com retinil palmitato no pós-parto imediato sobre os níveis de retinol do colostro. Rev Panam Salud Publica. 2007; 22: 51-4.

18. Ribeiro KDS, Araújo KF, Pereira MC, Dimenstein R. Avaliação dos níveis de retinol no colostro humano coletado no intervalo de 24 horas. J Pediatr. (Rio J.) 2007; 83: 377-80.

19. Dimenstein R, Nascimento THCR, Melo ILP, Ribeiro, KDS. Avaliação dos níveis de retinol no colostro humano e a sua relação com o estado nutricional materno em vitamina A. Rev Bras Med. (Rio de Janeiro) 2006; 65: 206-10.

20. Passos PAL. Carotenóides e vitamina A em colostro de mães de recém-nascidos pré-termo, atendidas no CAISM [dissertação]. Campinas: Universidade Estadual de Campinas, Faculdade de Engenharia de Alimentos; 1993.

21. Trigo MS. Retinol no leite humano: estudo em lactantes da área urbana do Recife-PE [tese]. São Paulo: Programa de Pós-Graduação em Ciência dos Alimentos da Universidade de São Paulo; 1988. 
22. Presta FMP. Composição do leite materno em vitamina A, carotenóides e vitamina E: relação com o estado nutricional e características maternas [dissertação]. Rio de Janeiro: Universidade Federal do Rio de Janeiro, Instituto de Química; 2001.

23. Oliveira MCC. Teores de minerais e vitamina A no leite humano em diferentes fases de lactação segundo variáveis maternas [dissertação]. Minas Gerais: Universidade Federal de Minas Gerais, Faculdade de Farmácia; 2003.

24. Mello Neto J. Influência de fatores nutricionais, obstétricos, socioeconômicos e demográficos nas concentrações de vitamina A, ferro, zinco e cobre no sangue e no leite maduro de doadores do banco de leite humano de Marília, SP [tese]. São Paulo: Faculdade de Saúde Pública da Universidade de São Paulo; 2005.

25. Newman V. Vitamin A and breastfeeding: a comparison of data from developed and developing countries. San Diego: Wellstart International; 1993.

26. Burri BJ. Beta-carotene and human health: a review of current research. Nutr Res. 1997; 17: 547-80.

27. Bahl R, Bhandari N, Wahed MA, Kumar GT, Bhan MK. Vitamin A supplementation of women postpartum and of their infants at immunization alters breast milk retinol and infant vitamin A status. J Nutr. 2002; 132: 3243-8.

28. Vinutha B, Mehta MN, Shanbag P. Vitamin A status of pregnant women and effect of post partum vitamin A supplementation. Indian Pediatr. 2000; 37: 1188-93.

29. Rice AL, Stoltzfus RJ, De Francisco A, Chakraborty J, Kjolhede CL, Wahed MA. Maternal vitamin A or betacarotene supplementation in lactating Bangladeshi women benefits mothers and infants but does not prevent subclinical deficiency. J Nutr. 1999; 129: 356-65.

30. Stoltzfus RJ, Hakimi M, Miller KW, Rasmussen KM, Dawiesah S, Habicht JP, Dibley MJ. High dose vitamin A supplementation of breast-feeding Indonesian mothers: effects on the vitamin A status of mother and infant. J Nutr. 1993; 123: 666-75.

31. Green MH, Green JB, Akohoue SA, Kelley SK. Vitamin A intake affects the contribution of chylomicrons vs. retinolbinding protein to milk vitamin A in lactating rats. J Nutr. 2001; 131: 1279-82.

32. Gebre-Medhin M, Vahlquist A, Hofvander Y, Uppsäll L, Vahlquist B. Breast milk composition in Ethiopian and Swedish mothers. I. Vitamin A and $\beta$-carotene. Am J Clin Nutr. 1976; 29: 441-51.

33. Barua S, Tarannum S, Nahar L, Mohiduzzaman M. Retinol and alfha-tocopherol content in breast milk of Bangladeshi mothers under low socio-economic status. Int J Food Sci Nutr. 1997; 48: 13-8.

34. Christian P, West KP Jr. Interactions between zinc and vitamin A: an update. Am J Clin Nutr. 1998; 68 (Suppl. 2): S 435-41.

35. Dijkhuizen MA, Wieringa FT, West CE, Muhilal. Zinc plus beta-carotene supplementation of pregnant women is superior to beta-carotene supplementation alone in improving vitamin A status in both mothers and infants. Am J Clin Nutr. 2004; 80: 1299-307.

36. Amine EK, Corey J, Hegsted DM, Hayes KC. Comparative hematology during deficiencies of iron and vitamin $\mathrm{A}$ in the rat. J Nutr. 1970; 100: 1033-40.

Recebido em 17 de julho de 2007

Versão final apresentada em 15 de dezembro de 2008

Aprovado em 18 de dezembro de 2008
37. Staab DB, Hodges RE, Metcalf WK, Smith JL. Relationship between vitamin A and iron in the liver. J Nutr. 1984; 114: 840-4.

38. Rosales FJ, Jang JT, Pinero DJ, Erikson KM, Beard JL, Ross AC. Iron deficiency in young rats alters the distribution of vitamin A between plasma and liver and between hepatic retinol and retinyl esters. J Nutr. 1999; 129: 1223-8.

39. Jang JT, Green JB, Beard JL, Green MH. Kinetic analysis shows that iron deficiency decreases liver vitamin A mobilization in rats. J Nutr. 2000; 130: 1291-6.

40. Oliveira JM, Michelazzo FB, Stefanello J, Rondó PHC. Influence of iron on vitamin A nutritional status. Nutr Rev. 2008; 66: 141-7.

41. Shatrugna V, Raman L, Uma K, Sujatha. Interaction between vitamin A and iron: effects of supplements in pregnancy. Int J Vitamin Nutrit Res. 1997; 67: 145-8.

42. Greer FR. Fat-soluble vitamin supplements for enterally fed preterm infants. Neonatal Netw. 2001; 20: 7-11.

43. Thomas MR, Pearsons MH, Demkowicz M, Chan IM, Lewis CG. Vitamin A and vitamin E concentration of the milk from mothers of pre-term infants and milk of mothers of full term infants. Acta Vitaminol Enzymol. 1981; 3: 13544.

44. Panpanich R, Vitsupakorn K, Harper G, Brabin B. Serum and breast-milk vitamin $\mathrm{A}$ in women during lactation in rural Chiang Mai, Thailand. Ann Trop Paediatr. 2002; 22 : 321-4.

45. Canfield LM, Clandinin MT, Davies DP, Fernandez MC, Jackson J, Hawkes J, Goldman WJ, Pramuk K, Reyes H, Sablan B, Sonobe T, Bo X. Multinational study of major breast milk carotenoids of healthy mothers. Eur J Nutr. 2003; 42: 133-41.

46. Meneses F, Trugo NMF. Retinol, beta-carotene, and lutein + zeaxanthin in the milk of Brazilian nursing women: associations with plasma concentrations and influences of maternal characteristics. Nutrit Res. 2005; 25: 443-51.

47. Patton S, Canfield LM, Huston GE, Ferris AM, Jensen RG. Carotenoids of human colostrum. Lipids. 1990; 25: 159-65.

48. Stoltzfus RJ, Habicht JP, Rasmussen KM, Hakimi M. Evaluation of indicators for use in vitamin A intervention trials targeted at women. Int J Epidemiol. 1993; 22: 1111-8.

49. Rice AL, Stoltzfus RJ, De Francisco A, Kjolhede CL. Evaluation of serum retinol, the modified-relative-doseresponse ratio, and breast-milk vitamin A as indicators of response to postpartum maternal vitamin A supplementation. Am J Clin Nutr. 2000; 71: 799-806.

50. De Pee S, Dary O. Biochemical indicators of vitamin A deficiency: serum retinol and serum retinol binding protein. J Nutr. 2002; 132 (Suppl.): S 2895-901.

51. Filteau SM, Morris SS, Abbott RA, Tomkins AM, Kirkwood BR, Arthur P, Ross DA, Gyapong JO, Raynes JG. Influence of morbidity on serum retinol of children in a community-based study in northern Ghana. Am J Clin Nutr. 1993; 58: 192-7.

52. Dancheck B, Nussenblatt V, Ricks MO, Kumwenda N, Neville MC, Moncrief DT, Taha TE, Semba RD. Breast milk retinol concentrations are not associated with systemic inflammation among breast-feeding women in Malawi. J Nutr. 2005; 135: 223-6. 